\title{
A Map-Based Visualization Tool To Support Tutors In E-Learning 2.0
}

\author{
Roberto Pirrone \\ Vincenzo Cannella \\ Giuseppe Russo \\ Universita' degli Studi Palermo Universita' degli Studi Palermo Universita' degli Studi Palermo \\ Viale delle Scienze Ed. 6 P $3 . \quad$ Viale delle Scienze Ed. 6 P $3 . \quad$ Viale delle Scienze Ed. 6 P 3. \\ 90128 Palermo (IT) \\ 90128 Palermo (IT) \\ 90128 Palermo (IT) \\ pirrone@unipa.it \\ cannella@dinfo.unipa.it \\ russoedinfo.unipa.it
}

\begin{abstract}
Web 2.0 regards essentially the social issues about the new usage of web applications, but participative web and user generated contents induce a new way to think about the design of the web applications themselves. This is particularly true in the field educational systems that are all web based applications. Many researchers are now devoted to study what is called e-learning 2.0 both as regards the technological issues in the field of computer science, and in relation to the impact of the web 2.0 social and psychological issues on the education process itself. One of the most crucial topics in e-learning 2.0 is the way to provide support to the teacher/tutor to avoid cognitive overload when he/she is monitoring the evolution group dynamics inside the class, and decides the proper strategies to ensure the pursuit of the learning goals. Map visualization is a good way to present information without cognitive overload. We present a map-based tool in support of the tutor that is an extension of our ITS called TutorJ. The tool allows a human tutor to have multiple map visualizations about the domain of the course, the social (forum-based) interaction between the students, and the amount of topics faced by each student. The paper reports a detailed description of the architecture of the tool, and a discussion about its relevance in the field of elearning 2.0.
\end{abstract}

\section{INTRODUCTION}

Web 2.0, in a few words, involves all the social issues regarding the use of web technology. In turn, new paradigms of using the web lead to a new concept of web applications [1] [2] as regards both their development and management. The major novelties of web 2.0 applications are about highly interconnected interactive contents, and the users' direct contribution to their creation. A more and more relevant part of the contents provided in several organizations' web sites are created and controlled by their users. This leads to network effects. This is a major revolution: new applications are more and more user-centric. Popular examples are YouTube or Facebook where users have the complete responsibility to define and publish their contents as codevelopers. Indeed, web 2.0 applications cover several fields such as higher education, government, and all the economic aspects of social networking.

A crucial aspect is related to contents visualization. The large amount of available data have to be presented in an appropriate manner. Visualization is an important aspect to face the information overflow that is a consequence of the huge number of contributions posted on the net. It is also very important to reach a good level of detail without loosing the perception of the whole available materials.
The definition of a new type of web applications requires a paradigm shift in traditional operations such as data collection and data analysis. Web 2.0 sites are very dynamic in the creation of new relations across their users and tools like forums, wikis and blogs are central in the process of dynamic content creation.

In particular, higher educational can benefit of new technologies in many ways. Learning activities are highly distributed, social and complex tasks and therefore the learning model has to provide solutions in this direction. As an examples wikis and forums are excellent tools to obtain cooperation inside a workgroup and between different ones.

A web 2.0 approach in designing educational tools, that is e-learning 2.0, requires a major effort from teachers and tutors to keep track and regulate the group dynamics and to offer personalized solutions to each learner. The presented work tries to address this issue with a social network [3] analysis tool that helps to explain social behavior and provides typical representation of social interactions. This is the first step in the evaluation process, while the second one is the need to produce hints and warning in particular situations. To this aim we propose a visual analysis tool able to offer different possible visualizations of collected data to help both students and tutors. Our tool is an extension of TutorJ an ITS proposed by some of the authors [4], which arranges all the course contents in a browsable pan-zoom map addressed by the concepts in the domain ontology that are related to the documents stored in the database. The map is able to cluster similar documents. TutorJ has been extended to allow gathering of the information related to the use of the document map. In turn, usage information is the source for creating different student maps representing the amount of documents read for each topic in the domain. Finally, forum analysis has been added providing information to display some social network maps where the relations between the learners arranged in discussion groups are shown. The same tool is used to display relation between the workgroups created by the tutor. The rest of the paper is organized as follows: section II presents briefly the state of the art of web 2.0 and learning environments with particular attention to visualization tools. The outline of the whole tool is presented in section III. Architectural issues are addressed in detail in section IV. Finally, section V reports conclusions and future work. 


\section{STATE OF THE ART}

Many Learning Management Systems or Learning Content Management Systems are focused on content delivery, management and distribution without taking the necessary care about the social aspects of learning. As stated in [5] a paradigm shift is needed in traditional pedagogical models. The most important changes have to be performed w.r.t. the central role of users instead of contents and materials. A distributed approach has to be preferred with a bottom-up growth of materials based on sharing.

The design of learning systems able to assist social groups in collaborative works has lead to a new generation of Virtual Learning Environments (VLE) where learning activities are performed through web 2.0 technologies: the e-learning 2.0 [6] [7].

According to the constructivist theories, learning cannot be transmitted but has to be constructed by the individual. The way a student learns can be more oriented to the discovery of new knowledge [8] or rather the social aspects of learning can be focused [9]. In the context of Educational Psychology a student able to define autonomously the way to increase her knowledge performs a self-regulated learning process [10]. One of the major pedagogical issues is related to the cognitive overload of students that arises as one the major problems for self-regulated learning in technology-enhanced learning environments[11].

An e-learning 2.0 environment takes a great advantage from visual representation of contents and social relations. The most used tool is the map. Maps are visualizations of relations between components (physical or abstract objects as ideas, relationships and so on). A simple example of a social network map is the contact map used in different sites and applications to show personal information of registered users. Another example is Chat Circle [12] developed by Sociable Media Group, is a visual abstract interface showing real time conversation in Internet.

Maps are powerful tools to show contents too. An example of this kind of interface is the Spiral map in [13] where documents are arranged on the basis of their publication date as a spiral. The WebSOM [14] is an example of document map built automatically by a neural network. This approach uses a Self-Organizing Map to cluster documents. Documents' displacement inside the map is arranged on the basis of their clustering. A similar approach is used in the KnowledgeSea map [15] a two dimensional map of educational resources. Each cell contains documents related semantically to each other. Documents located in cells connected directly are less similar, and so on. In [16] a map visualization is used to render different opinions in a forum used to score different products. Authors use Cytoscape [17] and Jung [18] to visualize different opinions.

\section{OUTLINE OF THE VISUALIZATION TOOL}

Our visualization tool is integrated in TutorJ, and shares the same three tiers architecture as the ITS (see fig. 1). The core functionality in TutorJ regards contents presentation with a browsable pan-zoom map where the learning paths built by the teacher/tutor or suggested by the ITS can also be drawn. The student can browse the documents freely or he/she can follow tutor's proposals. Such an approach has been inspired to the cognitivist theories of learning.

We extended the system to allow the student to interact with her colleagues, or with a tutor. Workgroups can be established by the tutor, or they can be built by the students autonomously. A group can be made by two or more students. Students in a group can interact exchanging messages through a forum, or cooperate to write a wiki.

The new system allows the tutor to monitor the students' activities through the use of social network maps or student maps. We developed some suitable information managers in the business level of the architecture to collect and analyze the logs of either student-system or student-student interactions. The presentation layer provides the map visualization of the information generated by the managers. A novel unified paradigm has been devised to render whatever kind of map, starting from a unique XML syntax to describe the structure and the relations between the items to be displayed at the highest level of abstraction.

Student maps show the documents studied by a learner, and the amount of time he/she spent in reading them. In this way, the tutor evaluates if a student perceived the topic under investigation as a difficult or interesting one. She has a global information about topics that have not deepened sufficiently. Finally, the tutor can analyze both the behavior of either the single student or an entire workgroup.

Social network maps show the relations between students in a group and/or between groups starting from the information collected in the analysis of their interactions in forums or wikis. It's depicted in the form of a graph where each node is clickable and provides information about the related student or group. Arcs represent the strength of a relation on the basis of the amount of communication streams that have been recorded between two subjects by the information manager.

Finally, maps can be useful to compare two different data sets. Maps can show the evolution of the state of the student while he/she is learning the domain. A sequence of maps can depict the states of the student in different moments. Moreover, the tutor can compare the students in a same study group, assessing commonalities and differences in their activities. A possible interaction scenario is the following: the tutor opens the social network map and clicks on two student nodes in the same group whose relation is particularly strong. He visualizes the two student maps and locates common areas, thus obtaining the information about the topic they're deepening together. The previous scenario involves a solely visual interaction, and avoids reading more and more lines of messages in the forum. The creation of difference maps and overlay visualization can be thought in this respect.

\section{THE ARCHITECTURE}

The software architecture of the proposed tool is arranged in three tiers (see Fig. 1). 


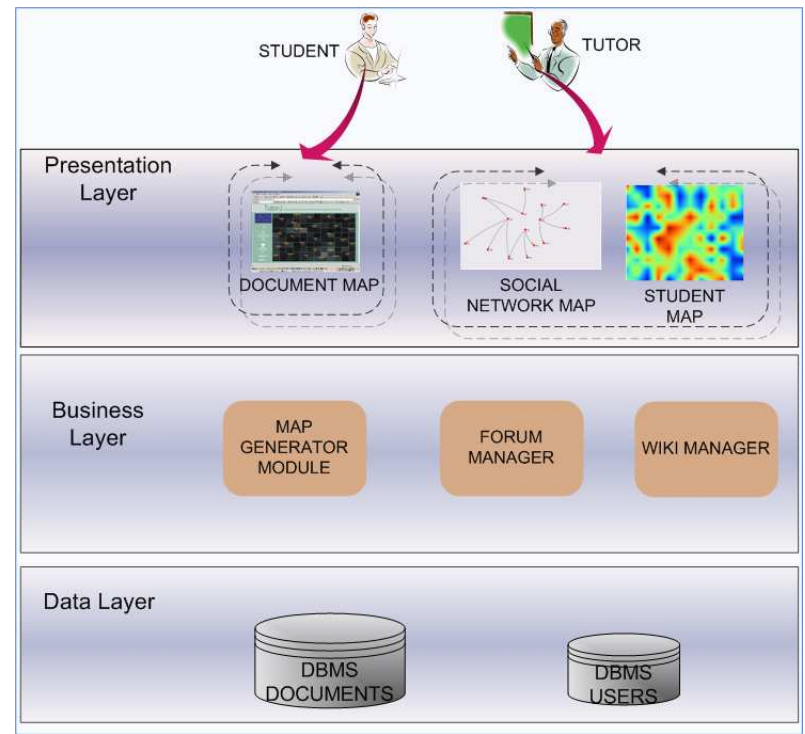

Fig. 1. The architecture of the visualization tool with the new modules in each layer.

The figure shows only the new modules added to the previous TutorJ implementation. At the highest level the presentation layer is able to show the different possible visualizations. The business layer manages and collects data from the interaction with different actors. The documents and the information about users are stored in the data layer.

\section{A. Data Layer}

This layer is the back-end of the whole system. It manages many kinds of data: learning materials, didactic proposals by the tutors, and logs of student-system and student-student interactions. Documents are analyzed and grouped according to the topic they deal with. Automatic cataloguing makes subject-based consulting very easy. It would be a hard task if performed manually. Students and tutors can enrich the set of documents. Students can create cooperatively new contents through wikis. The tutor can always add new learning materials. The $\log s$ of the interactions of the students trace their behavior, allowing an assessment of students' activities, or an analysis of the social dynamics in the groups.

Learning documents must be analyzed to be catalogued. To this aim, they are represented in a sub-symbolic fashion through the Latent Semantic Analysis [19] technique. Thanks to this technique, documents are represented as real vectors in a metric space. When two documents treat similar subjects, they are projected into near points in the hyperspace. In this way, clusters of documents dealing with common subjects can be found using a clustering technique. In particular, we have used a Self-Organizing Map [20], which is able to cluster points, and reduces the number of dimensions of the final space. The result is a bi-dimensional vectorial space where documents are represented as points, and clustered into similarity regions. The pipeline of the whole process is depicted in Fig. 2.

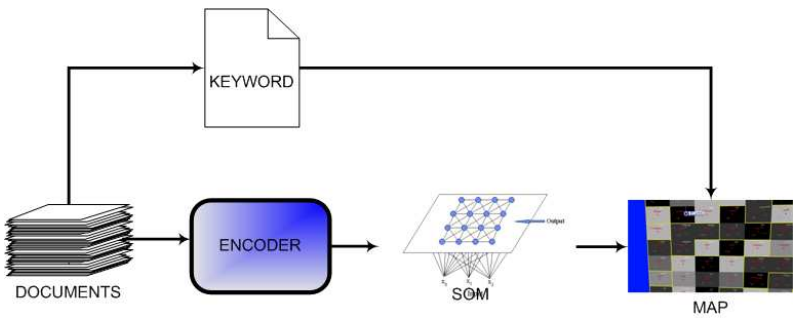

Fig. 2. The sequence of steps for the generation of a document map. The document map generator in the business layer is in charge to render the X3D document map starting from the data stored in the trained SOM, and from the list of keywords used to run LSA.

The data layer stores also the didactic proposals by the teachers/tutors that are displayed as learning paths in the document map at the presentation level .The logs of students' interactions are also recorded, to be shown to tutors. Finally, the layer manages all the data related to the social interactions between the students. It stores wikis, and forums. Moreover, it stores the participants to the workgroups and their interactions. In this way, the layer stores all the data needed to analyze the behavior of either a single student or a whole group.

\section{B. Business Layer}

In this layer we implemented the main modules to manage data and produce suitable interaction modalities with students and tutors. The new modules we added to the previous TutorJ implementation are the map generator module, the wiki manager and the forum manager. The forum manager and the wiki manager are devoted to manage and collect information deriving from social interaction between student and from collaborative writing of documents in the form of a wiki. These types of interaction are used to build the social network and give information to the tutors about the groups dynamics. The map generator module is able to retry the information regarding a particular interaction modality (browsing documents, analysis of social behaviors or analysis of the access to documents) and to convert them into a suitable visual map. This is the major novelty presented in this work. The information about students are used as inputs in the map generator module. The implemented tool has a unified representation formalism of input data that are converted in XML units and processed with proper tools to render the suitable map. In figure 3 the data flow to build all the presented maps is depicted.

The data are processed and transformed in an XML [21] meta-representation. After this initial phase the resulting $\mathrm{XML}$ is processed. Different maps are obtained according to proper XSLT transformations [22]. There are essentially two possible outputs: a X3D [23] format used to represent both the document and the student map and a graphML [24] format that is the input for the JUNG [18] framework and is used to represent the social interactions between students. The document map is used mainly by students while the student map and the social network map are 


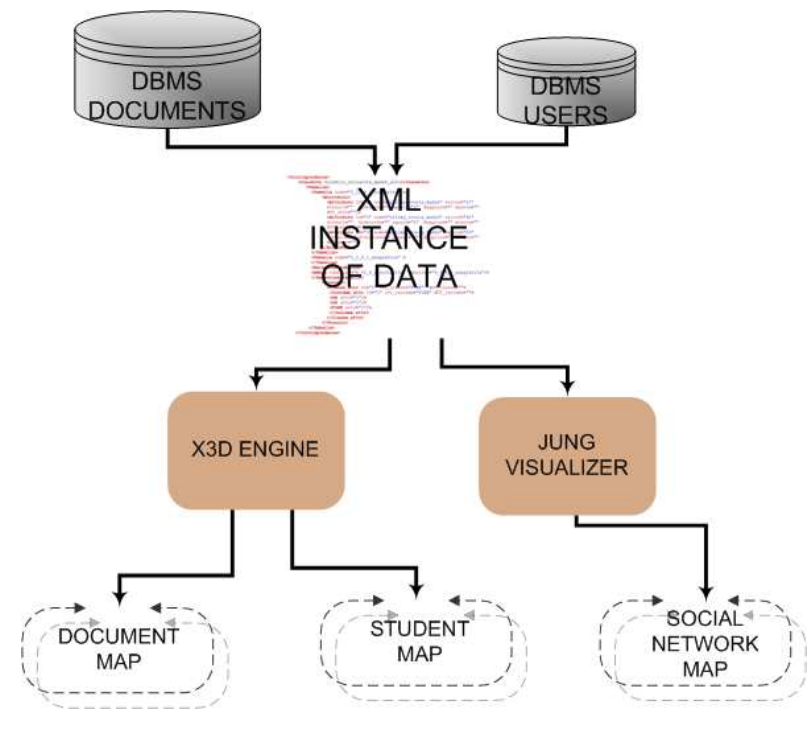

Fig. 3. The data flow in the map generator module. Required data are merged into a unique XML-based representation. Then the XML files are analyzed by an XSL processor and the corresponding rules are activated. This allows different representation of data.

used by tutors/teachers. In particular, when rendering the document map some suitable methods have been included in the XSLT transformation to collect data from the SOM and the list of keywords used to run LSA (see fig. 2). This approach allows the system to have a good level of scalability. New functionalities can be added simply by implementing the proper XSLT transformation. In our approach, a new visualization modality corresponds to a new system's usage scenario. Another advantage of the proposed approach is related to data hierarchy. If a single student requires his document map the system has to retrieve the XML node with the proper id, and just process it. If the information of the student's group are needed the system has to increase the level of node hierarchy in the XML file (every group is composed by a certain number of students).

\section{Presentation Layer}

The presentation layer allows the student to browse learning materials using a document map. Tutors make use of the social network map and the student map to support their decisions, while monitoring the students. In the document map, the regions obtained form clustering are placed in the space on the basis of the similarity of the topics covered in their documents. Clusters are as close as they're similar. Each region is associated to a set of more representative keywords, able to recall the common subjects of its documents. In this way, documents can be consulted easily using a subject-based research. The documents space is represented graphically as a starred sky where a document is a star, and a cluster of documents is depicted as a constellation. The main keyword associated to the cluster is the name of the corresponding constellation. This name is depicted on the map. The map is depicted in X3D (see Fig. 4).

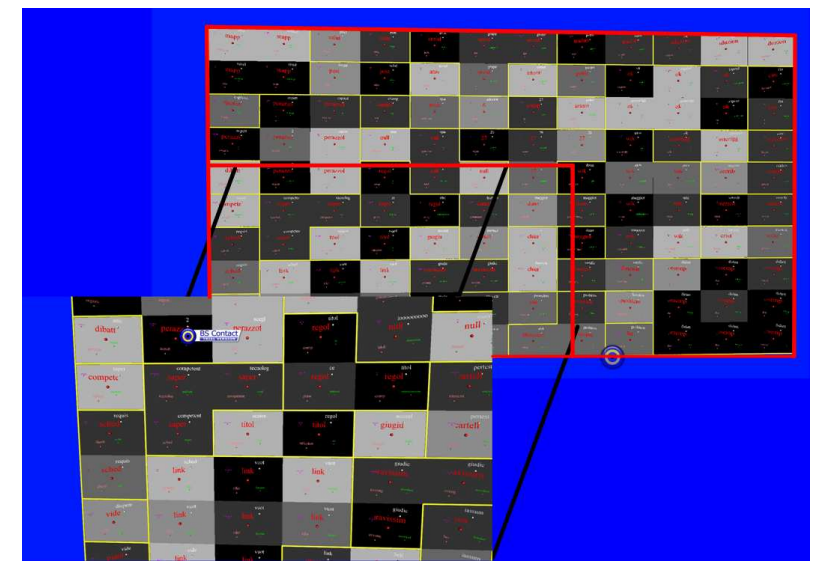

Fig. 4. An example of document map along with the first zooming level. Regions representing documents belonging to a particular subject are bounded by yellow lines. Red words are the keywords representing regions. The gray levels of an area in the map reflects the number of documents in that area.

The map is a Zooming User Interface. It can be zoomed in and out, and the user can shift up, down, left and right. Zooming in an area of the map allows to see that area in more detailed way. Less important keywords characterizing the documents are shown. Smaller stars appear in the scene. At the highest degree of zooming, the list of documents of the area is shown, and it's possible to click and read them. The document is open in a new tab. In this way, the student can go back to the map to select new documents to be opened together. All these clicks are recorded by the system, to model the student. The analysis of these data can be very informative. The system can evaluate if the student follows the learning path proposed by the tutor, or if she studied documents freely. In this case, it could be interesting to discover which documents the students has opened together in a single session. Finally, all these actions are recorded with a timestamp. In this way, the tutor knows how much time the student devoted to studying a documents.

Two different types of map have been defined to support tutors in their work. These maps are obtained analyzing the number of documents read by the student for each region of the maps of documents, or the total time spent reading the documents of each region. In the student map, these values are displaced as elevation values on a regular grid that is the same used for the document map. Elevations are interpolated to generate a continuous bi-dimensional surface, colored with a pseudo-color reflecting the height of the points. We used a classical temperature gradient palette where red indicates a very huge amount of time spent in reading documents, while blue is related to poorly accessed materials (see Fig. 5).

The map is a sort of planimetric map. It is generated as an image, and placed as texture on a X3D plane. This choice makes its rendering faster. The user can select her own palette, or link a particular palette to a specific type of data. She can also customize a palette. The obtained map can be superimposed to the documents map. In this case, 


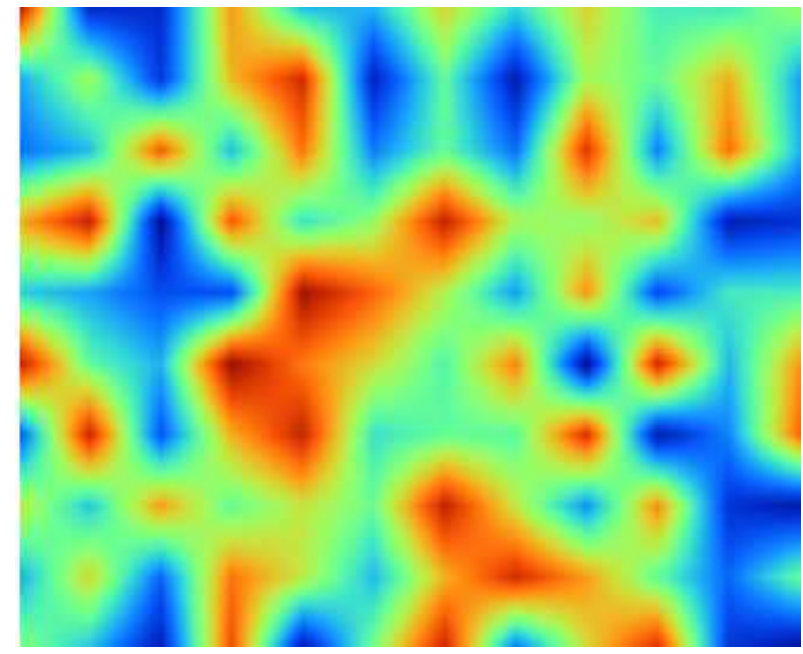

Fig. 5. An example of student map. It uses pseudo-color to show the amount of time spent in reading documents in a cluster.

the names of the clusters shown in the documents maps are written onto the student map. This provides the tutors with a detailed representation of the documents the student read actually. If the mouse is placed on a region of the map, a tooltip with summarizing data appears. Also this map is a zooming user interface. The user can zoom in and out the map, and when she click on a selected region the interface shows the detailed data for that region.

The tutor can analyze a whole group too. In this case, the system represents the group as a net, whose nodes represents the persons belonging to the group (see Fig. 6).

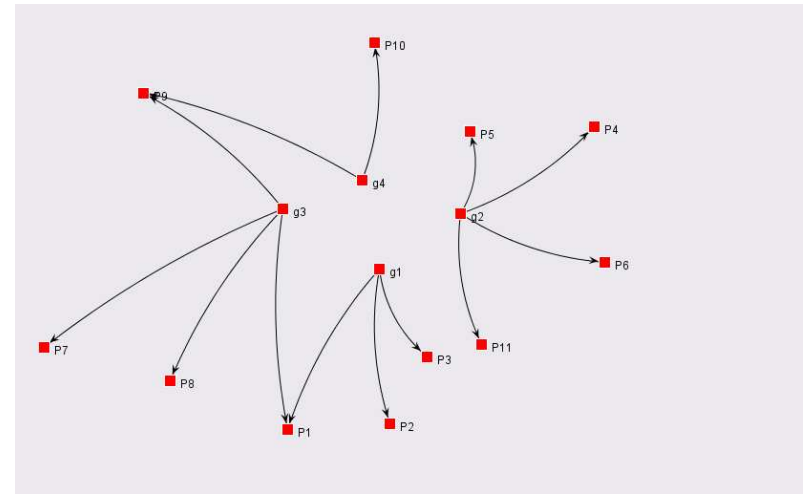

Fig. 6. A social network map. A class composition is represented by a graph. When the tutor clicks on a node, she can consult data regarding a student or a group. Many kinds of maps can be related to the same node according to different inquiries about the student/group.

The nodes of the members of a group are linked to a particular node representing the group. Arcs carry out the information related to the amount of communications between two nodes (students or groups). When the tutor clicks on a node of a person, she can view all the data regarding the single student. When she clicks on a group node, she can see the data regarding the whole group.
Similarly to the student maps, the tool can produce social network maps summarizing the data of the group, regarding all the documents read by the members of the group. As an alternative, the tutor can see distinct maps to compare different data sets, put side by side, or laying one over the others and switched one after the others. As a last chance, the tutor can see comparison maps, obtained from the pointby-point difference of two maps.

\section{CONCLUSIONS AND FUTURE WORKS}

A map based visualization tool to support tutor decision making, while monitoring the students' activities has been presented. The tool extends the architecture of TutorJ an ITS already presented by some of the authors. The work is motivated by the need to avoid tutor's cognitive overload in the analysis of social interactions between the students and/or their learning activities. It uses web 2.0 technologies to allow purely visual interaction with the huge amount of information deriving from the logs of the student-student and studentsystem interactions. Our implementation confirms the thesis stating that social use of the web, that is web 2.0, induces the creation of a new generation of web applications to manage information overload, and in turn such applications rely heavily on the web 2.0 leading to an enhancement in the technology. Future works involve the porting of the whole TutorJ implementation on a well known open source elearning platform that is OLAT [25] and the implementation of the knowledge maps. Such maps are derived from the structure of the wikis produced by the learner on some specific topic, and can be compared with a fragment of the domain ontology proposed by the teacher to discover how the student is acquiring the concepts in a particular topic, and the logical relations between them and rest of the domain.

\section{REFERENCES}

[1] T. O'Reilly, "O'reilly network: What is web 2.0," September 2005. [Online]. Available: http://www.oreillynet.com/lpt/a/6228

[2] G. Vossen and S. Hagemann, Unleashing Web 2.0. - From Concepts to Creativity. Burlington, MA: Morgan Kaufman, 2007.

[3] I. Liccardi, A. Ounnas, R. Pau, E. Massey, P. Kinnunen, S. Lewthwaite, M.-A. Midy, and C. Sarkar, "The role of social networks in students' learning experiences," in ITiCSE-WGR '07: Working group reports on ITiCSE on Innovation and technology in computer science education. New York, NY, USA: ACM, 2007, pp. 224-237.

[4] R. Rizzo, G. Pilato, and R. Pirrone, "A KST-Based System for Student Tutoring," Applied Artificial Intelligence, vol. 22, no. 4, pp. 283-308, 2008.

[5] M. A. Chatti, M. Jarke, and D. Frosch-Wilke, "The future of elearning: a shift to knowledge networking and social software," IJKL, vol. 3, no. 4/5, pp. 404-420, 2007.

[6] S. Downes, "E-learning 2.0," eLearn Magazine, October 2005. [Online]. Available: http://elearnmag.org/subpage.cfm?section= articles $\backslash \& \backslash \# 38$; article $=29-1$

[7] C. Ullrich, K. Borau, H. Luo, X. Tan, L. Shen, and R. Shen, "Why web 2.0 is good for learning and for research: principles and prototypes," in WWW '08: Proceeding of the 17th international conference on World Wide Web. New York, NY, USA: ACM, 2008, pp. 705-714.

[8] J. S. Bruner, On Knowing: Essays for the Left Hand. Burlington, MA: Cambridge, Mass.: Belknap Press of Harvard University Press., 1962.

[9] L. S. L. S. Vygotsky, Mind in society. Burlington, MA: Harvard University Press, Cambridge, MA, 1978.

[10] B. J. Zimmermann, Theories of Self-Regulated Learning and Academic Achievement: An Overview and Analysis. Lawrence Erlbaum Associates, 2001, ch. 1, pp. 1-37. 
[11] OECD, "Learning for Tomorrows World First Results from PISA 2003." [Online]. Available: http://www.oecd.org/document/55/0,3343, en_32252351_32236173_33917303_1_1_1_1,00.html

[12] F. B. Viégas and J. S. Donath, "Chat circles," in CHI '99: Proceedings of the SIGCHI conference on Human factors in computing systems. New York, NY, USA: ACM, 1999, pp. 9-16.

[13] "Spiralinterface," http://rhizome.org/spiral/.

[14] S. Kaski, K. Lagus, T. Honkela, and T. Kohonen, "Statistical Aspect of the WEBSOM System in Organizing Document Collections," Computing Science and Statistics, vol. 29, pp. 281-290, 1998.

[15] P. Brusilovsky and R. Rizzo, "Using maps and landmarks for navigation between closed or open corpus." The New Review of Hypermedia and Multimedia, 2003.

[16] C. Kaiser, B. S. Tiwana, and F. Bodendorf, "Bridging the gap between qualitative and quantitative analysis of opinion forums," Web Intelligence and Intelligent Agent Technology, IEEE/WIC/ACM International Conference on, vol. 1, pp. 120-126, 2008.

[17] M. S. Cline, M. Smoot, E. Cerami, A. Kuchinsky, N. Landys, C. Workman, R. Christmas, I. Avila-Campilo, M. Creech, B. Gross, K. Hanspers, R. Isserlin, R. Kelley, S. Killcoyne, S. Lotia, S. Maere, J. Morris, K. Ono, V. Pavlovic, A. R. Pico, A. Vailaya, P. L. Wang, A. Adler, B. R. Conklin, L. Hood, M. Kuiper, C. Sander, I. Schmulevich, B. Schwikowski, G. J. Warner, T. Ideker, and G. D. Bader, "Integration of biological networks and gene expression data using cytoscape." Nat Protoc, vol. 2, no. 10, pp. 2366-2382, 2007. [Online]. Available: http://dx.doi.org/10.1038/nprot.2007.324

[18] "JUNG: Java Universal Network/Graph Framework." [Online]. Available: http://jung.sourceforge.net

[19] T. Landauer, P. Foltz, and D. Laham, "An Introduction to Latent Semantic Analysis," Discours Processes, vol. 25, pp. 259-284, 1998

[20] T. Kohonen, Self-Organizing Maps. Berlin, Heidelberg: Springer, 1995.

[21] T. Bray, J. Paoli, C. Sperberg-McQueen, and E. Maler, "Extensible markup language (xml), 1.0 second edition," W3C Recommendation, 2006. [Online]. Available: http://www.w3.org/TR/ 2006/REC-xml-20060816/

[22] M. Kay, "XSL transformations (XSLT) Version 2.0," W3C," W3C Recommendation, Jan. 2007, http://www.w3.org/TR/2007/REC-xslt2020070123/.

[23] "X3D International Specification Standards." [Online]. Available: http://www.web3d.org/x3d/specifications/x3d/

[24] "The GraphML File Format." [Online]. Available: http://graphml. graphdrawing.org/index.html

[25] The OLAT team at the University of Zurich, "OLAT - The Open Source LMS.” [Online]. Available: http://www.olat.org/website/en/ $\mathrm{html} /$ index.html 